\title{
A Systematic Review of the Statistical Methodology Used in Establishing the Link Between Climate Factors and HFMD Incidence
}

\author{
Nurmarni Athirah Abdul Wahid ${ }^{a}$, Jamaludin Suhailaa,b,*, Haliza Abd. Rahman ${ }^{a}$ \\ ${ }^{a}$ Department of Mathematical Sciences, Faculty of Science, Universiti Teknologi \\ Malaysia, 81310 Johor Bahru, Johor, Malaysia; ${ }^{b}$ UTM Centre of Industrial and Applied \\ Mathematics (UTM-CIAM), Universiti Teknologi Malaysia, 81310 Johor Bahru, Johor, \\ Malaysia
}

${ }^{*}$ For correspondence: suhailasj@utm.my

Received: 15 January 2021 Accepted: 15 October 2021

(C) Copyright Wahid. This article is distributed under the terms of the Creative Commons Attribution License, which permits unrestricted use and redistribution provided that the original author and source are credited.

\begin{abstract}
Hand, foot, and mouth disease (HFMD) is a common infectious disease caused by two main viruses, namely Coxsackievirus A16 and Human Enterovirus 71. It has been a significant public health disease and a substantial burden all over the world since 1969. Prior studies have shown that climate factors are significantly associated with HFMD cases by using various statistical methods. Therefore, this study aims to review the scientific studies related to climate and HFMD and hence, address the analytical techniques used. This study only includes quantitative studies from peer-reviewed and original papers published in international and national journals from the years 1957 to 2020. In total, there were 522 articles identified; however, there were only 29 studies that linked climate change and HFMD. Based on the articles reviewed, the modelling analysis technique, which includes the Generalized Linear Model (GLM), the Generalized Additive Model (GAM), and the Generalized Additive Mixed Model (GAMM), represents the most popular analysis in identifying the association between HFMD and climate factors. The temperature and humidity showed the greatest impact on the occurrence of HFMD, and the suitable incubation period for all climatic factors was not more than three weeks.
\end{abstract}

Keywords: HFMD, climate change, generalized linear model, generalized additive model.

\section{Introduction}

Hand, foot, and mouth disease, which is known as HFMD, has been one of the most rapidly spreading viral infections all over the world since 1957. HFMD was first introduced clinically in Canada and New Zealand in the year 1957. This viral disease can be easily transmitted from person to person. HFMD is a syndrome that usually affects children; however, it also infects adolescents occasionally [1]. Various serotypes of Enterovirus cause HFMD. The majority of HFMD cases were caused by Coxsackie Virus A16 and Enterovirus 71 (EV-71).

According to the Ministry of Health Malaysia [2], HFMD begins with a fever, which is a temperature of 38.2 degrees Celsius, a feeling of being unwell, and less appetite. After the fever starts for one or two days, painful sores will develop in the throat, mouth, and tongue, or herpangina. It usually begins with a rash or spots containing fluid with small blemishes about 3 to $7 \mathrm{~mm}$ in diameter, often in the back of 
the mouth, and they will be in the hands, wrists, feet, and diaper areas. Most people who get infected by HFMD will have mild illness or no symptoms at all, but a small proportion of cases can be moresevere [3]. The severe forms of HFMD may lead to permanent damage to the body or even death. Thus, preventive measures and awareness against this disease are some of the effective ways to prevent it from spreading.

Since the first isolation in California in 1969, EV-71 has caused isolated outbreaks of HFMD as well as serious neurological diseases such as meningitis, encephalitis, and acute flaccid paralysis (AFP) [4]. In recent years, this infectious disease has been a massive burden throughout Eastern and South-eastern Asia, which has caused significant public health concerns worldwide. In 1975, HFMD appeared in Bulgaria; 750 people were infected, 149 people had paralyzes, and 44 people died [5]. In the late 1990s, two widespread community outbreaks related to EV-71 infection occurred in Sarawak, Malaysia [6]. In 1998, the most massive and most severe EV-71 epidemic occurred in Taiwan, which affected mainly children. HFMD was getting even worse with neurological complications and pulmonary edema, and there were 78 children dying because of this infectious disease [7]. There were large-scale HFMD outbreaks in the years 2008 and 2009 in Mainland China that led to almost five hundred thousand and 1.2 million reported cases, respectively [8].

Climate change has recently been shown to have a significant impact on health diseases such as haemorrhagic fever, dengue disease, acute respiratory infection, and HFMD [9-12]. Previous studies have revealed that HFMD incidence was significantly associated with climate factors including rainfall, temperature, humidity, wind speed, and others in temperate countries. Thus, this study aims to perform a systematic review to identify the statistical methodology used to establish the relationship between climate factors and HFMD incidence.

\section{Methodology}

\section{Search Strategy}

A report from the years 1957 to 2020 found an association between HFMD incidence and climate is reviewed systematically. It is based on the scientific findings derived from the statistical method as well as laboratory and field studies on HFMD. A literature search was conducted in October 2018 using electronic databases such as Scopus, PubMed, Science Direct, Web of Science, and the World Health Organization publication databases to obtain information on the impact of climate variables on HFMD transmission. This study searched each of the databases using the combinations of keywords including "HFMD," "climate," "temperature," "hand, foot, and mouth disease," "relative humidity," "rainfall," "weather," and "wind speed". However, this study limited the literature search to journal articles that have been published in English and are available in full-text or pdf. The references and citations of the materials identified were checked twice to ensure that all relevant articles were included.

\section{Selection Criteria}

In this study, the articles are selected from the search results based on criteria chosen for detailed consideration. First, to obtain authoritative information, this review only includes the peer-reviewed and original papers published in international and national journals from the year 1957 to 2020 . Next, all the selected articles had to include larger geographical areas and climate factors such as temperature, relative humidity, wind speed, rainfall, and so on. Besides, the statistical analytical method with at least one climate variable was also considered. Finally, the review includes quantitative studies based on statistical purposes (climate-based) because qualitative studies used an entirely different analytical and research design approach. The findings and discussion focus on the establishment of the link between climate factors and HFMD incidence. This study also provides recommendations for future research directions towards model parameters and predictions of climate change on HFMD infection.

Figure 1 shows a summary of the strategies, processes, and results of the literature search that includes the four steps, which are identification, screening, eligibility, and final. In the first step of identification, 
the records were identified through the database searching of scientific publications specifically on HFMD incidence and climate factors. The inclusion criteria for this process include the peer-reviewed and original papers that were published in international and national journals from 1957 to 2020. There are 587 articles found from the databases in the identification process of this study. Following that, only 522 articles were selected based on the inclusion criteria in the screening process. However, 447 articles were removed after viewing the titles and abstracts. The selected articles must include a larger geographical area of study, using climate data, and a statistical analytic method with at least one climate factor. In the eligibility step, there were only 75 articles that included only quantitative studies which had been considered in detail. However, only 29 reviews strictly met the criteria of the study, which related to climate change and HFMD infectious disease.

\section{Results}

\section{Statistical Methods Used in HFMD Cases and Climate Factors}

There are fifteen studies that used weekly confirmed and reported cases of HFMD data, five studies used monthly cases, and nine studies used daily reported cases. Previous studies have used various types of analytical approaches, which include correlation analysis, Geographically Weighted Regression (GWR), generalized modelling techniques, time-series models, distributed lag non-linear models (DLNM), spatial models, and Classification and Regression Tree Model (CART). Figure 2 illustrates the percentage of statistical methodology used by the selected studies. The generalized modelling approaches were the most widely used methods among the researchers since it indicates the highest numbers, with 16 studies, followed by the spatial and time-series models with 4 and 3 studies, respectively. Three types of generalized linear model were considered, including the Generalized Linear Model (GLM), Generalized Additive Model (GAM), and Generalized Additive Mixed Model (GAMM). However, the analysis shows that most of the studies used the GAM approach with 9 studies, and only 1 study used the GAMM approach. Each of the statistical methods used by the selected studies is explained in greater detail in the following subsection.

\section{Correlation Analysis}

Correlation analysis is a standard useful statistical parameter that describes the degree of relationship between two variables. Before proceeding with the principal analysis, some researchers used correlation analysis as their first step in analysis to avoid the existence of multicollinearity, which may lead to a wrong conclusion. A correlation analysis was performed to assess the relationship between incidences of HFMD and weather in Singapore [13]. The study indicates that weather variations may affect the transmission of HFMD in Singapore. Besides, there are few studies that used Spearman's rank correlation to examine the association between HFMD cases and each meteorological parameter before they proceed with regression analysis [14-18].

\section{Geographically Weighted Regression}

Geographically weighted regression has been applied in various studies to examine the associations between selected factors and HFMD incidence. GWR has been used in exploring the spatial relationships of HFMD occurrences with different conditions that require climatic variables and the density of the child population in China [19]. They announced that the GWR model could be used to geographically discern the relationship between the infection and other explanatory variables.

\section{Generalized Modelling Techniques}

Generalized modelling techniques such as the generalized linear model (GLM) and generalized additive model (GAM) have been extensively applied to assess the influence of meteorological factors on HFMD incidence. GLM enables linear modelling concepts to be applied to a larger range of response types, such as count data or binary response. The GLM method is a flexible generalization of ordinary linear regression that allows the response variables to have various versions of error distribution other than the normal distribution. For example, a study in Japan [20] used the GLM analysis to examine the intense 
connection between weekly HFMD cases with temperature and humidity. They then used a negative binomial regression to account for over-dispersion in data, and the model contains up to a sixth harmonic with the Fourier terms. After accounting for possible variability due to weather fluctuations and inter-year differences, the study resulted in a rise in the number of HFMD cases as temperatures and relative humidity rose. The result provides sufficient evidence that climatic factors have a significant impact on the occurrence of HFMD infections.

Besides, a negative binomial regression that accounts for over-dispersion issues has been used to explore the relationship between HFMD and meteorological variables in China [17]. The study noticed that there are problems of collinearity, as both average temperature and air pressure demonstrate a high association between both. Thus, they performed two different models in which the first one consists of an average temperature but without air pressure. The second model, by comparison, consists of air pressure but with no average temperature. Both models contained additional climatic influences, including relative humidity and wind speed. They strongly believe that HFMD has a major impact on weather conditions. A study in Malaysia [21] compared six different models that were estimated using the Poisson and negative binomial GLM. This approach was applied to identify the association between HFMD incidence and temperature with the effect of lag time. Based on the six models with different lag values of temperature, the study found that a negative binomial GLM with temperature at 2 weeks' lag time provided the smallest value of AIC and the best reduction of scaled deviance. Therefore, they conclude that a two-week lag in temperature is an important climate factor to explain the HFMD incidence in Selangor, Malaysia.

Besides, a Poisson GLM combined with a distributed lag non-linear model (DNLM) has been applied to estimate the interaction between extreme precipitation and childhood HFMD and to investigate whether the correlation differed between urban and rural areas [22]. The study showed that an increase in cases of childhood HFMD was correlated with extreme precipitation, and children living in urban areas were more susceptible to the impact of extreme precipitation on HFMD compared to those living in rural areas. Accordingly, they provide verifiable evidence that the change in climatic conditions significantly influences the increase in HFMD.

A semi-parametric extension of the GLM approach was conducted by some researchers, called the generalized additive model (GAM). For these statistical approaches, the only basic assumption is that the functions are additive and the elements are smooth. This approach is useful in identifying exposureresponse relationships for many data types, especially in exploring non-parametric relationships and also providing a flexible means of managing non-linear covariate effects [23]. A study in Guangzhou, China [24], for example, used these methods to examine the impact of meteorological influences on the occurrence of HFMD in children. They used a penalized smoothing spline to adjust the long-term trend and seasonal cycle of six degrees of freedom per annum in everyday morbidity. As a result, the researcher provides proof that meteorological factors may play a significant role in HFMD distribution.

A related analysis was performed in Rizhao, China, which attempted to examine the short-term impact of meteorological influences on adolescent HFMD aged between 0 and 18. They used GAM with log link and penalized smoothing splines approaches to achieve their study goals [15]. However, the research strategy was contradicted by a study in Guangzhou, China [24] in which the authors stratified the analysis by sex (male, female), age group (less than or equivalent to 2 years, 2 to 18 years of age), and child occupation, whether scattered or schooling. Scattered children here mean pre-school children that have not been to kindergarten or school. As a result, they stated that climate factors such as temperature, relative humidity, and sunshine hours were statistically important for adolescent HFMD in Rizhao.

Besides, the GAM approach was also used to assess the relationship between latitude as well as meteorological variables and HFMD for the island in East Asian countries [25]. They used GAM with a Gaussian distribution with a cubic smoothing spline method for meteorological variables and a linear term for the latitude in model fitting. They implied that the most important explanatory factor associated 
with the timing and magnitude of HFMD occurrence was latitude. Thus, the weather conditions and geographical position might play a major role in influencing HFMD outbreaks. A GAM with smoothing splines was applied to measure the average temperature, relative humidity, sunshine period, precipitation, and wind speed from 16 climate centres situated in 16 major cities and provinces in South Korea [18]. In their analysis, time lags of up to two weeks in climate variables were considered. Based on the analysis, they noticed that the HFMD rate rises along with average temperature and relative humidity up to some points with $18^{\circ} \mathrm{C}$ and $65 \%$, respectively, and then decreases with increasing temperature and humidity subsequently. They demonstrate that only the average temperature and relative humidity in South Korea had a significant connection with the HFMD cases.

Another study used a GAM with a negative binomial family to identify the correlations between weekly occurrences of HFMD and mean temperature, relative humidity, and Baidu Index (BDI) in two different cities in Guangdong Province, China, Shantou, and Shenzen [3]. In their study, they used the BDI as the internet search index, as it was the most commonly used in China. They believe that the GAM method is useful in defining the relationship of exposure-response for many kinds of data, particularly in exploring the non-parametric relationship. The study claims that both internet search engines and climate variables can improve HFMD event prediction.

Similar research in Guangzhou, China, used meteorological factors and the search engines to query data by combining them in their study [26]. By using historical HFMD events, internet-based search engine query data, and meteorological factors, the study aimed to build an effective monitoring model of HFMD. By performing several steps, they expanded the conventional GAM model to a network-based GAM model. In the first phase, they computed the Pearson correlation between cities. To compare the time series of HFMD incidence in Guangzhou with the other cities in Guangdong Province, they found five cities with the highest correlation with Guangzhou. Then, by assigning weights to the search indices of the five towns, the researcher used the correlation to create a new variable. They combined the HFMD cases in the previous two weeks into a model as an auto-regression term due to the potential delay in the formal report of the monitoring system. In the last phase, they combined the HFMD cases of Guangzhou, the search pattern, the weighted search pattern of the other five cities, meteorological factors, seasonal patterns, and the population factors. The study shows that combining data from search queries and meteorological factors will help improve the estimation of the occurrence of HFMD.

The generalized additive mixed model (GAMM) is an extension of the GAM in which this approach takes into consideration the auto-correlation between the repeated measures by adding the random effects and differentiating between the subject and the variability within the subject [27]. In eight major cities in Guangdong, China, the GAMM has been applied to investigate the climate impact on paediatric HFMD cases and analyse the possible effect modification by geographic factors [16]. To monitor the seasonality and long-term pattern of HFMD incidence, the GAMM with a natural cubic spline of time with 6 degrees of freedom per year was employed. They claim that this approach offers an excellent capability to manage residual autocorrelation and leads to robust estimates and standard errors. They indicated that geographic latitude and longitude were closely linked to climatic factors.

\section{Time Series Models}

Time-series forecasting models are one of the most common statistical approaches among researchers to examine the performance of meteorological factors on the occurrences of HFMD. A Seasonal Autoregressive Integrated Moving Average (SARIMA) model is a valuable tool for interpreting and applying disease control and prevention surveillance data [28]. The SARIMA method was used to determine the association of climate variables with HFMD epidemics among children in Zhengzhou, Henan, China [29]. They stated that SARIMA models (Box and Jenkins models) have the flexibility to monitor the autocorrelation of the time series data. They found that the predictive power for SARIMA models of HFMD and EV-71 increased after the addition of the mean atmospheric temperature at lag two weeks. 
An autoregressive integrated moving average model with external variables (ARIMAX) was applied to explore whether the addition of external predictors such as the BDI and temperature could boost the risk prediction of HFMD [30]. The researchers used the time series analysis to allow the establishment of robust and effective ARIMAX models with the correct validity level, which matches the provincial-wide HFMD incidence. Consequently, they conclude that using the ARIMAX model and introducing the BDI helps to greatly enhance the estimation of HFMD incidence. Finally, they proposed that using an ARIMAX multivariate model would offer better prediction over the univariate model.

\section{Distributed Lag Non-Linear Models}

A few studies in China used a distributed lag non-linear model (DLNM) in determining the significant relationship between climate variables and HFMD cases. A Poisson distribution DLNM has been applied to analyse the non-linear lag effects of daily climatic factors and the levels of particulate matter with an aerodynamic diameter of 10 micrometres $\left(\mathrm{PM}_{10}\right)$ [31]. They used a DLNM approach as it provides a thorough representation of a non-linear exposure-response relationship and prevents collinearity issues among lagging exposure variables compared to other models. Besides, this statistical approach was also used to explore the effects of humidex on HFMD in children under the age of 15 and its heterogeneity across social-economic status and age groups [32]. The humidex is a comprehensive index known as a meteorological index that incorporates temperature and humidity impacts and is directly comparable to dry temperature in degrees Celsius. The researchers decided to use DLNM with the distribution of quasiPoisson since this approach allows the predictor-HFMD relationships to be tested simultaneously at different lags without co-linearity problems.

\section{Spatial Models}

Some researchers have also used spatial modelling approaches to investigate the association between cases of HFMD and climate factors. In contrast to other forms of modelling, such as cross-sectional and time series models, the spatial panel data models are more insightful and contain less collinearity and more variation between the variables [33]. In addition, the panel data offers greater availability of degrees of freedom and thus improves the estimation's effectiveness. These statistical methods are typically used to analyse data containing time-series observations of specific spatial units, such as regions, states, and countries [34].

On the other hand, this method could address data with spatial dependence and allow both spatial and temporal heterogeneity to be considered [35]. Three spatial models were developed in a study in Shandong Province, China, including the model of fixed effects, the model of the spatial lag panel, and the model of spatial error. The study noticed that the spatial lag model was more effective than the other models and thus revealed that the spatial panel data model used in their analysis is more useful for depicting the association between the occurrence of HFMD and climate factors. Additionally, this approach was used in a study to analyse the relationship between meteorological factors as well as the incidence of HFMD according to the different climates of mainland China [36]. The study is divided into four classes by various types of environments, namely subtropical monsoon climate, temperate monsoon climate, moderate continental climate, and plateau mountain climate. The results showed that the model of spatial lag is more appropriate, where both the spatial autoregressive coefficient and the spatial autocorrelation coefficient are positive and statistically relevant. This suggests that spatial units are positively correlated with the incidence of surrounding spatial units and unmet variables.

In contrast, a spatiotemporal zero-inflated model was applied to examine the data with excessive zeros and to assess the efficacy of the zero-inflated impact between spatiotemporal zero-inflated models and conventional models [37]. This approach is a mixture of the spatiotemporal and zero-inflated models. The researchers specify the environmental risk factors for HFMD, taking into consideration both climate and socioeconomic aspects. They developed four models to test zero-inflated model efficiency, including the Poisson model, the negative binomial, the zero-inflated Poisson model, and the zero-inflated negative binomial model. By comparing the models, they observed that the zero-inflated negative binomial model for HFMD cases was the best explained. 


\section{Classification and Regression Tree Model (CART)}

In a few studies, the Classification and Regression Tree Model (CART) was used to classify the relationship between the incidence of HFMD and climatic factors. This method was performed to determine the threshold effects of climate factors on HFMD cases in China [38]. The researchers have developed a suite of two spatiotemporal CART models, including fitting a tree to a high HFMD risk and the incidence. A study in three separate areas in Gansu, China, used CART to explore the interactive non-linear impact between climate factors and HFMD instances [39]. They used CART to discover the threshold value that had the best potential to split nodes in the optimal trees. The analysis indicated that CART offers much clearer information on the non-linear interaction of HFMD cases with climate variables.

\section{Impact of Climate Factors on HFMD}

In recent years, there has been a rising number of literature on the impact of climate influences on HFMD incidence in several countries and areas. In modelling these issues, the choice of climate variables is an important problem that needs to be considered in each analysis. The common climate variables chosen by the researchers are mean temperature, minimum temperature, maximum temperature, relative humidity, wind speed, hours of sunshine, atmospheric pressure, and rainfall. Furthermore, Figure 3 depicts a summary of the significant climate factors influencing HFMD incidence as determined by the selected studies. The analysis reveals that the majority of the studies found that temperature is significantly related to HFMD incidence compared to the other climate factors. Humidity indicates the second important factor in HFMD incidence, followed by wind speed and rainfall. However, only one study implies that there is an association between visibility and HFMD incidence. Additionally, the relation of important and other variables with the incidence of HFMD is summarised in (See Table 1).

\section{Discussion}

Several statistical approaches were reviewed in this study, such as correlation analysis, generalized modelling (GLM, GAM, GAMM), time series modelling, spatial model, and CART model. The most common approaches used to investigate the relationship between climate factors and HFMD occurrences are generalized modelling techniques. This modelling approach permits the distribution of response variables to come from an exponential family and offers the availability to deal with any form of data set. Also, it is easy to understand how the results are influenced by each predictor. In this case, it is easy to understand and perceive the important effects of each climate variable on HFMD. However, some limitations of the GLM and GAM approaches were recognized. This includes the GLM limits to account for the non-linearity effect between variables and requires independent response variables [40]. Even though the GAM approach relaxes the linearity assumption of GLM, this approach is still limited to the fact that the response variables must be independent [41]. Since the HFMD series is normally timeseries data, autocorrelation among the repeated series might exist, and thus, the independent assumption of GLM and GAM will be violated. Therefore, the GAMM methods seem the best approach to be used in identifying the association between HFMD incidence and climate factors as they can account for the issue of autocorrelation in the data sets.

Besides, although the association between climate variables and HFMD incidence was already established in several studies, the findings are inconsistent. Several researchers in China have stated that HFMD has a positive effect on climate factors such as temperature $[15,16,19,21,24,30,32,39,42$, $43,44]$. Nevertheless, other studies from various regions of China have shown that temperature does not have a significant influence on HFMD $[3,37,45]$. Studies in Japan, Vietnam, and South Korea have shown that the number of HFMD cases increased significantly with the increasing average temperature and relative humidity $[18,20,46]$. The minimum temperature was found to be a risk measure for HFMD morbidity in Singapore compared to other climate variables [47]. According to a study in South Korea, as the temperature and humidity rise above $18^{\circ} \mathrm{C}$ and $65 \%$, respectively, the HFMD transmission decreases 
as people become uncomfortable being outside and reduce outdoor activities [18]. Moreover, a study in China documented that when the temperature is above $24.03^{\circ} \mathrm{C}$ and the relative humidity is less than $60.9 \%$ during non-autumn seasons, the chance of a high risk of HFMD will be $82.35 \%$ [30].

Other than temperature, the factors that caused the increasing cases of HFMD were wind speed and rainfall. Both climate variables showed a positive correlation with HFMD cases [36], whereas a study in Guangzhou [40] found that there was no link between wind speed and rainfall towards the occurrence of HFMD. In some studies, other climate factors, such as atmospheric pressure, have also affected the incidence of HFMD. The atmospheric pressure distribution exhibited the opposite of the distribution of HFMD cases [24]. In Southern China, the same findings were recorded in which one hPa rise in atmospheric pressure corresponds to a $7.53 \%$ decrease in the amount of HFMD incidence [17]. The finding contradicts a study issued in Mainland, China, in which they found that the atmospheric pressure provides a positive correlation with the climate type of subtropical monsoons on HFMD occurrences [36].

According to a report in Mainland, China [37], the rise in daylight hours would increase the incidence of HFMD. This result contradicts research in Rizhao, China, which stated that there was an adverse effect on the daily period of sunshine $[15,36]$. This might be because the duration of daylight in the environment could influence the survival and transmission of those viruses [48]. The hours of daylight will control the movement of children as it would encourage children to spend less time outdoors as the duration of daylight is shorter. This scenario will therefore minimise the risk of any virus being transmitted, such as HFMD.

Other than that, several researchers found out that rainfall is one of the climate factors that affect HFMD occurrences. A study in thirty-one provinces of the mainland, China specified that HFMD was inversely correlated with monthly rainfall [36]. Fortunately, a study in Vietnam noted that there was a clear positive correlation between rainfall and the incidence of HFMD in which, for every rise in the rain, the relative risk of HFMD will rise. They also considered the Southern Oscillation Index (SOI) as a climate influence, and they found that the variables greatly influenced the rise of HFMD [46].

Furthermore, the delay time effect of climatic factors on HFMD incidence is also essential. Due to the incubation period for enteroviruses and the possible delay in parents' knowledge and responsiveness to children's clinical signs, one week's lag time may describe HFMD activities well [3]. Temperature has a positive effect on HFMD incidence at a five-day lag [15, 22], one-week lag [30,42], two-week lag [14,21], one-to-two-week lag [47], two-to-three-week lag [29], and three-week lag [46]. Besides, a few studies have reported that relative humidity has been correlated with HFMD at lags of one week [42], two weeks [14], and three weeks [46]. Moreover, a two-week delayed effect on wind speed had a significant positive effect on the occurrence of HFMD [14,29]. Research in Guangzhou, China observed that four to eight days of rainfall was significantly associated with HFMD [24], while in Rizhao, China, a study noticed that daylight hours had an adverse impact on the occurrence of HFMD at lag three to four days [15]. Thus, it provides ample evidence that the optimal incubation period is no longer than three weeks for all climatic factors.

According to the evidence from the articles reviewed, climate conditions play an important role in the occurrences of HFMD, and this depends on the geographical location and region. Most studies have shown that temperature contributes to a major impact on HFMD and relative humidity falls to the second factor. 


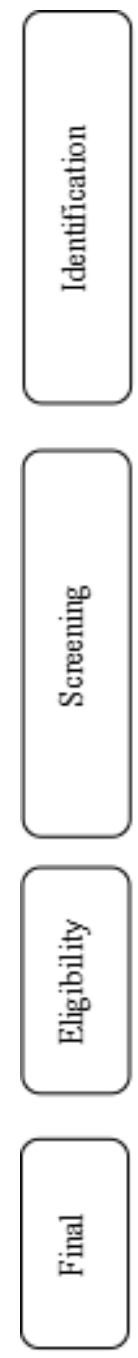

Records identified through database searching Scientific publications: HFMD and climate factors

Keywords: HFMD, climate, temperature, rainfall, relative humidity, wind speed, hand, foot, and mouth disease

Inclusion criteria: Peer-reviewed and original papers published in international and national journals from 1957-2020

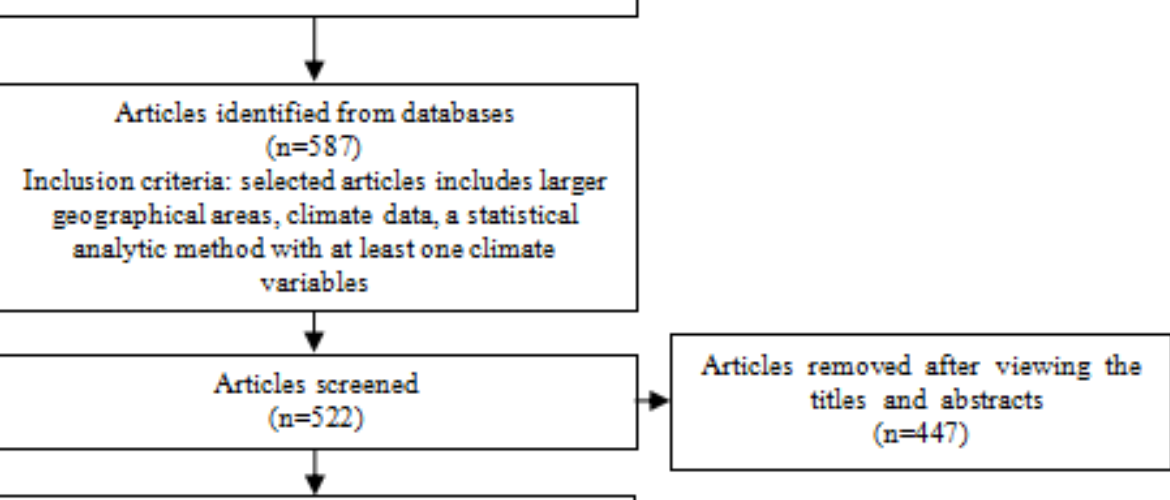

Articles identified for full-text review $(n=75)$

Inclusion criteria: selected articles includes quantitative studies based on statistical methods

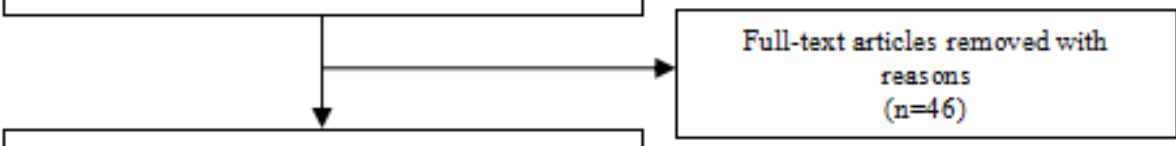

Articles included for this study $(n=29)$

Figure 1. Summary of strategies, process, and results of the literature search

Table 1. Significant variables on HFMD in selected studies 


\begin{tabular}{|c|c|c|c|c|c|c|c|c|c|c|c|}
\hline \multirow{2}{*}{$\begin{array}{l}\text { Author/ } \\
\text { Year }\end{array}$} & \multirow{2}{*}{$\begin{array}{l}\text { Resolution } \\
\text { of variables }\end{array}$} & \multicolumn{9}{|c|}{ Significant Climate Variable on HFMD } & \multirow{2}{*}{$\begin{array}{l}\text { Significant } \\
\text { Additional } \\
\text { Variables }\end{array}$} \\
\hline & & Temp & $\begin{array}{c}\text { Min } \\
\text { Temp }\end{array}$ & $\begin{array}{l}\text { Max } \\
\text { Temp }\end{array}$ & Rainfall & Humidity & $\begin{array}{c}\text { Sunshine } \\
\text { hours }\end{array}$ & $\begin{array}{c}\text { Atmospheric } \\
\text { pressure }\end{array}$ & $\begin{array}{l}\text { Wind } \\
\text { speed }\end{array}$ & Visibility & \\
\hline $\begin{array}{l}\text { Ma et al. } \\
(2010)\end{array}$ & Weekly & $\bullet$ & & & & $\bullet$ & & & $\bullet$ & & - \\
\hline $\begin{array}{l}\text { Hii et al. } \\
\text { (2011) }\end{array}$ & Weekly & $\bullet$ & & & $\bullet$ & & & & & & - \\
\hline $\begin{array}{l}\text { Leong et al. } \\
\text { (2011) }\end{array}$ & Weekly & $\bullet$ & & & & & & $\bullet$ & $\bullet$ & & - \\
\hline $\begin{array}{l}\text { Onozuka \& } \\
\text { Hashizume } \\
(2011)\end{array}$ & Weekly & $\bullet$ & & & & $\bullet$ & & & & & Age \\
\hline $\begin{array}{l}\text { Hu et al. } \\
(2012)\end{array}$ & Monthly & $\bullet$ & & & & - & & & $\bullet$ & & $\begin{array}{c}\text { Child } \\
\text { population } \\
\text { density }\end{array}$ \\
\hline $\begin{array}{l}\text { Huang et al. } \\
(2013)\end{array}$ & Weekly & $\bullet$ & & & & $\bullet$ & & & & & - \\
\hline $\begin{array}{l}\text { Chen et al. } \\
(2014)\end{array}$ & Daily & $\bullet$ & & & $\bullet$ & $\bullet$ & & & & & - \\
\hline $\begin{array}{l}\text { Lee et al. } \\
(2015)\end{array}$ & Weekly & & & & & $\bullet$ & & & $\bullet$ & $\bullet$ & $\begin{array}{l}\text { Geographical } \\
\text { location }\end{array}$ \\
\hline $\begin{array}{l}\text { Wu et al. } \\
\text { (2014) }\end{array}$ & Daily & $\bullet$ & & & & $\bullet$ & $\bullet$ & & & & - \\
\hline $\begin{array}{l}\text { Cheng et al. } \\
(2014)\end{array}$ & Daily & & & & $\bullet$ & & & & & & - \\
\hline $\begin{array}{l}\text { Feng et al. } \\
(2014)\end{array}$ & Weekly & $\bullet$ & & & & & & & & & - \\
\hline $\begin{array}{l}\text { Li et al. } \\
(2014)\end{array}$ & Daily & $\bullet$ & & & & $\bullet$ & & $\bullet$ & $\bullet$ & & - \\
\hline $\begin{array}{l}\text { Liao et al. } \\
(2015)\end{array}$ & Weekly & & & & & $\bullet$ & & & $\bullet$ & & - \\
\hline $\begin{array}{l}\text { Wang et al. } \\
(2015)\end{array}$ & Monthly & $\bullet$ & & & & $\bullet$ & & & $\bullet$ & & - \\
\hline $\begin{array}{l}\text { Wei et al. } \\
(2015)\end{array}$ & Daily & $\bullet$ & $\bullet$ & $\bullet$ & & & & & & & - \\
\hline $\begin{array}{l}\text { Guo et al. } \\
\text { (2016) }\end{array}$ & Daily & $\bullet$ & & & & $\bullet$ & & & & & - \\
\hline $\begin{array}{l}\text { Kim et al. } \\
(2016)\end{array}$ & Weekly & $\bullet$ & & & & $\bullet$ & & & & & - \\
\hline $\begin{array}{l}\text { Du et al. } \\
(2016)\end{array}$ & Monthly & $\bullet$ & & & & $\bullet$ & & & & & - \\
\hline $\begin{array}{l}\text { Huang et al. } \\
(2016)\end{array}$ & Daily & $\bullet$ & & & & $\bullet$ & & & & & - \\
\hline $\begin{array}{l}\text { Zhang et al. } \\
(2016)\end{array}$ & Daily & & & & & & & & & & Humidex \\
\hline $\begin{array}{l}\text { Thanh } \\
(2016)\end{array}$ & Weekly & $\bullet$ & & & $\bullet$ & $\bullet$ & & & & & - \\
\hline $\begin{array}{l}\text { Wang et al. } \\
(2016)\end{array}$ & Monthly & $\bullet$ & & & $\bullet$ & $\bullet$ & $\bullet$ & $\bullet$ & $\bullet$ & & - \\
\hline $\begin{array}{l}\text { Du et al. } \\
(2017)\end{array}$ & Weekly & $\bullet$ & & & & & & & & & Baidu Index \\
\hline $\begin{array}{l}\text { Huang \& } \\
\text { Wang (2018) }\end{array}$ & Weekly & $\bullet$ & & & $\bullet$ & $\bullet$ & & & & & - \\
\hline $\begin{array}{l}\text { Xiao et al. } \\
(2017)\end{array}$ & Daily & $\bullet$ & & & $\bullet$ & & & & & & - \\
\hline $\begin{array}{l}\text { Gou et al. } \\
(2018)\end{array}$ & Weekly & $\bullet$ & & & & $\bullet$ & & & $\bullet$ & & - \\
\hline $\begin{array}{l}\text { Song et al. } \\
(2018)\end{array}$ & Monthly & $\bullet$ & & & & $\bullet$ & $\bullet$ & & & & Socioeconomic \\
\hline $\begin{array}{l}\text { Chen et al. } \\
(2019)\end{array}$ & Weekly & $\bullet$ & & & & $\bullet$ & & & & & BDI \\
\hline $\begin{array}{l}\text { Wahid et al. } \\
(2020)\end{array}$ & Weekly & $\bullet$ & & & & & & & & & - \\
\hline
\end{tabular}

*Temp: Temperature; Min Temp: Minimum Temperature; Max Temp: Maximum Temperature 


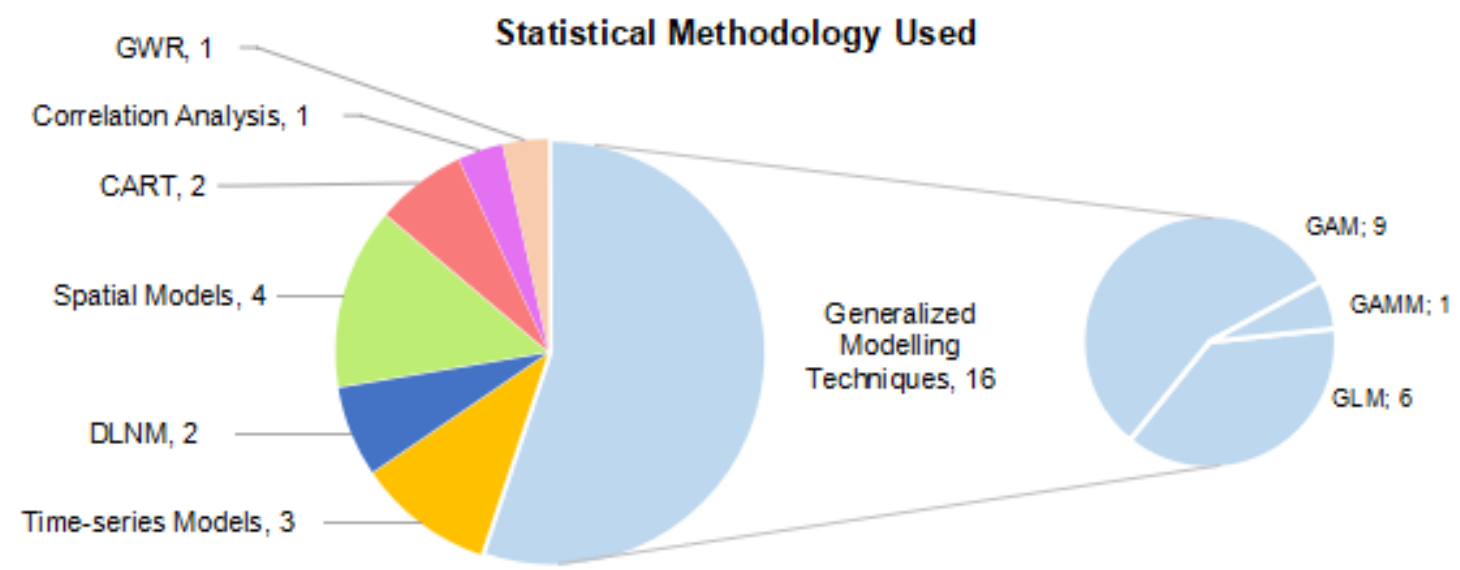

Figure 2. Summary of statistical methodology used by the selected studies

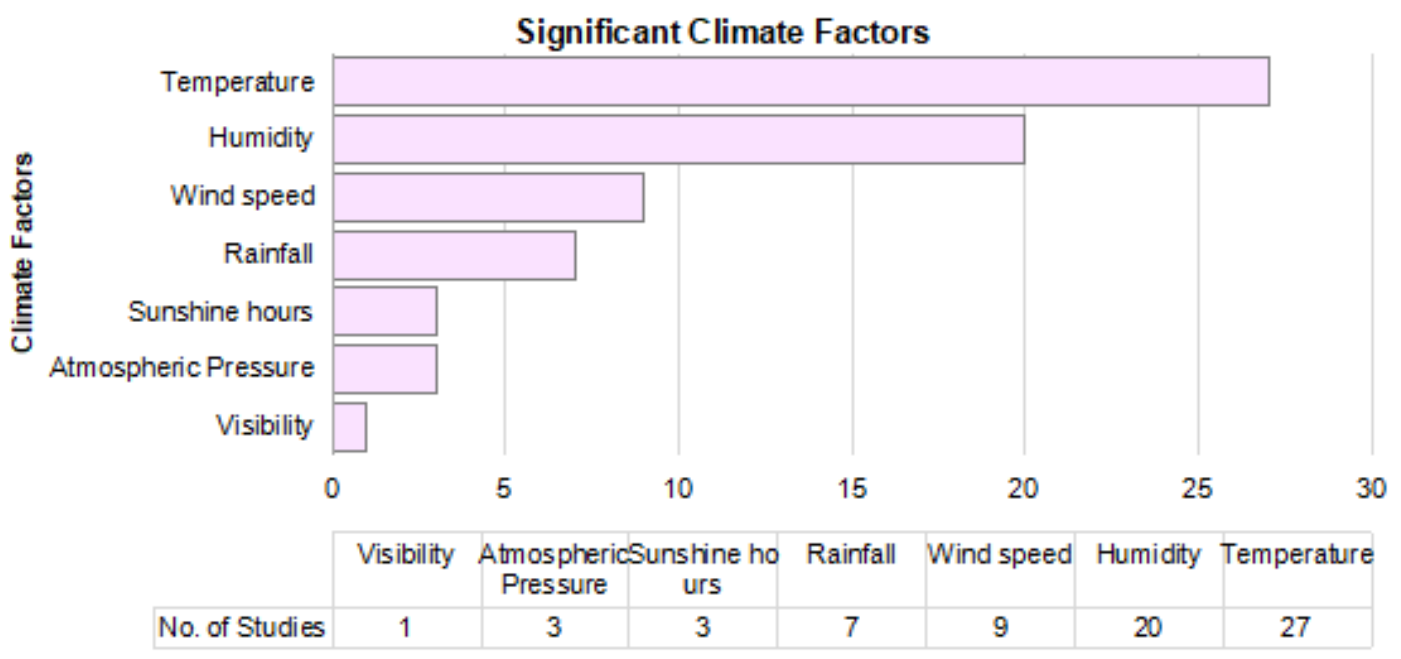

Figure 3. Summary of significant climate factors

\section{Conclusion}


This study reviewed the statistical methods used in identifying the relationship between HFMD incidence and climate factors. The method of generalized modelling approaches, including the GLM, GAM, and GAMM, indicates the most common approaches that have been applied to examine these related issues. However, the GAMM approach presents the best method since it can address the autocorrelation problems among the repeated series in the HFMD data sets. Many studies have succeeded in developing predictive models of HFMD based on climate relationships. However, the findings on the interaction between HFMD and climate influences were not consistent. It relies on the study's country or geographical position. The most important influences on the occurrence of HFMD are temperature and relative humidity. Nonetheless, at specific time lags, these variables are predictive. Other factors related to HFMD incidence, such as the age of the person infected, child population density, geographical location, search engine query data, socioeconomic status, and knowledge, attitudes, and practises (KAP) factors, should be included in future analysis. When using models as predictive tools for the occurrence of HFMD, there may be several limitations. In certain research, the concept of spatial stationarity is difficult to maintain. In local regions with the same conditions, the impact of the factors of interest on HFMD will be more similar. In contrast, the outcome would be different in the local areas whose requirements differ significantly. In addition, there was a study just using spatial data, in which spatial data alone does not provide the analysis of the temporal kinetics of HFMD incidence. Meanwhile, the use of temporal data alone does not allow for high-risk areas to be identified. Thus, studies should include both spatial and temporal factors in the study to enhance the efficiency of the final result and better predict HFMD incidence.

Other than that, the HFMD data could be unreported in hospitals and clinics because of the individual disease seriousness and the variations in the level of local healthcare services. Furthermore, because reported cases of HFMD vary over time and geography, HFMD data may be exaggerated or underreported. This data is influenced by the description of cases, the reporting of sub-clinical cases, as well as the availability of public health systems. Therefore, it is essential to consider all these factors in order to provide a complete report on the incidence of HFMD. To achieve good results and to reduce the detrimental impacts of climate change on the incidence of HFMD, there are several guidelines for future studies. The five important highlighted guidelines are as follows:

1) For good HFMD prevention and control initiatives, existing disease surveillance and monitoring systems incorporated into climate-sensitive conditions need to be strengthened;

2) Emphasize adaptation policies and strategies for vulnerable populations, and improve the effectiveness of the public health system in dealing with climate change in specific regional contexts;

3) In HFMD research, advanced spatiotemporal modelling interventions are required;

4) Consider other inputs in the model analysis, such as population growth, poverty, culture, population immunity, as well as behaviour and activities;

5) Increase the networking of HFMD issues between ecologists, sociologists, microbiologists, bio-statisticians, and epidemiologists.

Overall, this research concludes with ample proof that HFMD is vulnerable to climate variability and change. This study believes that it is essential to develop a different approach to HFMD quantitative modelling that is consistent with long-term climate data and other factors. This would therefore advance projections of climate impacts on HFMD cases.

\section{Conflicts of interest}

The authors declares that there is no conflict of interest regarding the publication of this paper.

\section{Funding statement}


This work was financially supported by by the Ministry of Higher Education under the Fundamental Research Grant Scheme FRGS/1/2020/STG06/UTM/02/3 (5F311) and Research University Grant with vote no: QJ130000.3854.19J58 and Zamalah UTM Scholarship under Universiti Teknologi Malaysia.

\section{Acknowledgments}

The authors would like to express their greatest appreciation to the Ministry of Health, Malaysia, for providing the HFMD data and the Malaysian Meteorological Department for providing the climatic data. This work was supported by the Ministry of Higher Education under the Fundamental Research Grant Scheme FRGS/1/2020/STG06/UTM/02/3 (5F311) and Research University Grant with vote no: QJ130000.3854.19J58 and Zamalah UTM Scholarship under Universiti Teknologi Malaysia.

\section{References}

[1] Melnick, J.L. 1984. Enteroviruses. In Viral infections of humans (pp.187-251). Springer, Boston, MA.

[2] Ministry of Health Malaysia. 2012. Zoonosis Sector. Department of Control Disease. Hand, Foot, and Mouth Disease (HFMD): Nasihat Kesihatan (Advisory) HFMD. Retrieved from http://www.moh.gov.my/index.php/pages/view/193.

[3] Chen, S., Liu, X., Wu, Y., Xu, G., Zhang, X., Mei, S., Zhang,Z., O'Meara, M., O'Gara, M.C., Tan, X. \& Li, L. 2019. The application of meteorological data and search index data in improving the prediction of HFMD: A study of two cities in Guangdong Province, China. Science of The Total Environment 652: 1013-1021.

[4] Schmidt, N.J., Lennette, E.H. \& Ho, H.H. 1974. An apparently new enterovirus isolated from patients with disease of the central nervous system. Journal of Infectious Diseases 129(3): 304309.

[5] Shindarov, L.M., Chumakov, M.P., Voroshilova, M.K., Bojinov, S., Vasilenko, S.M., Iordanov, I., Kirov, I.D., Kamenov, E., Leshchinskaya, E.V., Mitov, G. \& Robinson, I.A. 1979. Epidemiological, clinical, and pathomorphological characteristics of epidemic poliomyelitis-like disease caused by enterovirus 71. Journal of hygiene, epidemiology, microbiology, and immunology 23(3): 284-295.

[6] Chan, L.G., Parashar, U.D., Lye, M.S., Ong, F.G.L., Zaki, S.R., Alexander, J.P., Ho, K.K., Han, L.L., Pallansch, M.A., Suleiman, A.B. \& Jegathesan, M. 2000. Deaths of children during an outbreak of hand, foot, and mouth disease in Sarawak, Malaysia: clinical and pathological characteristics of the disease. Clinical infectious diseases 31(3): 678-683.

[7] Ho, M., Chen, E.R., Hsu, K.H., Twu, S.J., Chen, K.T., Tsai, S.F., Wang, J.R. \& Shih, S.R. 1999. An epidemic of enterovirus 71 infection in Taiwan. New England Journal of Medicine 341(13): 929-935.

[8] Wang, J.F., Guo, Y.S., Christakos, G., Yang, W.Z., Liao, Y.L., Zhong-Jie, L.I., Xiao-Zhou, L.I., Lai, S.J. \& Chen, H.Y. 2011. Hand, foot and mouth disease: spatiotemporal transmission and climate. International journal of health geographics 10(1): 1-10.

[9] Husam, I.S., Abuhamad, A.A.B., Zainudin, S., Sahani, M. \& Ali, Z.M. 2017. Feature selection algorithms for Malaysian dengue outbreak detection model. Sains Malaysiana 46(2): 255-265.

[10] Joshi, Y.P., Kim, E.H. \& Cheong, H.K. 2017. The influence of climatic factors on the development of hemorrhagic fever with renal syndrome and leptospirosis during the peak season in Korea: an ecologic study. BMC infectious diseases 17(1): 1-11.

[11] Santos, D.A.D.S., Azevedo, P.V.D., Olinda, R.A.D., Santos, C.A.C.D., Souza, A.D., Sette, D.M. \& Souza, P.M.D. 2017. The relationship of climate variables in the prevalence of acute respiratory infection in children under two years old in Rondonópolis-MT, Brazil. Ciência \& Saúde Coletiva 22: 3711-3722.

[12] Wang, Y., Yim, S.H.L., Yang, Y. \& Morin, C.W. 2020. The effect of urbanization and climate change on the mosquito population in the Pearl River Delta region of China. International Journal 
of Biometeorology 64(3): 501-512.

[13] Leong, P.F., Labadin, J., Abd Rahman, S.B. \& Juan, S.F.S. 2011. Quantifying the relationship between the climate and hand-foot-mouth disease (HFMD) incidences. In 2011 Fourth International Conference on Modeling, Simulation and Applied Optimization (pp. 1-5). IEEE.

[14] Ma, E., Lam, T., Wong, C. \& Chuang, S.K. 2010. Is hand, foot and mouth disease associated with meteorological parameter?. Epidemiology \& Infection 138(12): 1779-1788.

[15] Wu, H., Wang, H., Wang, Q., Xin, Q. \& Lin, H. 2014. The effect of meteorological factors on adolescent hand, foot, and mouth disease and associated effect modifiers. Global health action 7(1): 24664.

[16] Guo, C., Yang, J., Guo, Y., Ou, Q.Q., Shen, S.Q., Ou, C.Q. \& Liu, Q.Y. 2016. Short-term effects of meteorological factors on pediatric hand, foot, and mouth disease in Guangdong, China: a multi-city time-series analysis. BMC infectious diseases 16(1): 524.

[17] Li, Y., Zhang, J. \& Zhang, X. 2014. Modeling and preventive measures of hand, foot and mouth disease (HFMD) in China. International journal of environmental research and public health 11(3): 3108-3117.

[18] Kim, B.I., Ki, H., Park, S., Cho, E. \& Chun, B.C. 2016. Effect of climatic factors on hand, foot, and mouth disease in South Korea, 2010-2013. PloS one 11(6): e0157500.

[19] Hu, M., Li, Z., Wang, J., Jia, L., Liao, Y., Lai, S., Guo, Y., Zhao, D. \& Yang, W. 2012. Determinants of the incidence of hand, foot and mouth disease in China using geographically weighted regression models. PloS one 7(6): e38978.

[20] Onozuka, D. \& Hashizume, M. 2011. The influence of temperature and humidity on the incidence of hand, foot, and mouth disease in Japan. Science of the Total Environment 410: 119-125.

[21] Wahid, N.A.A., Suhaila, J. \& Sulekan, A. 2020. Temperature Effect on HFMD Transmission in Selangor, Malaysia. Sains Malaysiana, 49(10): 2587-2597.

[22] Cheng, J., Wu, J., Xu, Z., Zhu, R., Wang, X., Li, K., Wen, L., Yang, H. \& Su, H. 2014. Associations between extreme precipitation and childhood hand, foot and mouth disease in urban and rural areas in Hefei, China. Science of the Total Environment 497: 484-490.

[23] Hastie, T. \& Tibshirani, R. 1995. Generalized additive models for medical research. Statistical methods in medical research 4(3): 187-196.

[24] Chen, C., Lin, H., Li, X., Lang, L., Xiao, X., Ding, P., He, P., Zhang, Y., Wang, M. \& Liu, Q. 2014. Short-term effects of meteorological factors on children hand, foot and mouth disease in Guangzhou, China. International journal of biometeorology 58(7): 1605-1614.

[25] Lee, C.C.D., Tang, J.H., Hwang, J.S., Shigematsu, M. \& Chan, T.C. 2015. Effect of meteorological and geographical factors on the epidemics of hand, foot, and mouth disease in island-type territory, east Asia. BioMed research international 2015.

[26] Huang, D.C. \& Wang, J.F. 2018. Monitoring hand, foot and mouth disease by combining search engine query data and meteorological factors. Science of The Total Environment 612: 12931299.

[27] Wood, S.N. 2017. Generalized additive models: an introduction with R. CRC press.

[28] Luz, P.M., Mendes, B.V., Codeço, C.T., Struchiner, C.J. \& Galvani, A.P. 2008. Time series analysis of dengue incidence in Rio de Janeiro, Brazil. The American journal of tropical medicine and hygiene 79(6): 933-939.

[29] Feng, H., Duan, G., Zhang, R. \& Zhang, W. 2014. Time series analysis of hand-foot-mouth disease hospitalization in Zhengzhou: establishment of forecasting models using climate variables as predictors. PloS one 9(1): e87916.

[30] Du, Z., Xu, L., Zhang, W., Zhang, D., Yu, S. \& Hao, Y. 2017. Predicting the hand, foot, and mouth disease incidence using search engine query data and climate variables: an ecological study in Guangdong, China. BMJ open 7(10): e016263.

[31] Huang, R., Bian, G., He, T., Chen, L. \& Xu, G. 2016. Effects of meteorological parameters and PM10 on the incidence of hand, foot, and mouth disease in children in China. International journal of environmental research and public health 13(5): 481.

[32] Zhang, Z., Xie, X., Chen, X., Li, Y., Lu, Y., Mei, S., Liao, Y. \& Lin, H. 2016. Short-term effects of meteorological factors on hand, foot and mouth disease among children in Shenzhen, China: 
non-linearity, threshold and interaction. Science of the Total Environment 539: 576-582.

[33] Elhorst, J.P. 2009. Spatial panel data models. In the Handbook of Applied Spatial Analysis, Fischer, MM, and A. Getis.

[34] Deng, T., Huang, Y., Yu, S., Gu, J., Huang, C., Xiao, G. \& Hao, Y. 2013. Spatial-temporal clusters and risk factors of hand, foot, and mouth disease at the district level in Guangdong Province, China. PloS one 8(2): e56943.

[35] Wang, H., Du, Z., Wang, X., Liu, Y., Yuan, Z., Liu, Y. \& Xue, F. 2015. Detecting the association between meteorological factors and hand, foot, and mouth disease using spatial panel data models. International Journal of Infectious Diseases 34: 66-70.

[36] Wang, C., Cao, K., Zhang, Y., Fang, L., Li, X., Xu, Q., Huang, F., Tao, L., Guo, J., Gao, Q. \& Guo, $X .2016$. Different effects of meteorological factors on hand, foot and mouth disease in various climates: a spatial panel data model analysis. BMC infectious diseases 16(1): 233.

[37] Song, C., He, Y., Bo, Y., Wang, J., Ren, Z. \& Yang, H. 2018. Risk assessment and mapping of hand, foot, and mouth disease at the county level in mainland China using spatiotemporal zeroinflated Bayesian hierarchical models. International journal of environmental research and public health 15(7): 1476.

[38] Du, Z., Zhang, W., Zhang, D., Yu, S. \& Hao, Y. 2016. The threshold effects of meteorological factors on hand, foot, andmouth disease (HFMD) in China, 2011. Scientific reports 6: 36351.

[39] Gou, F., Liu, X., He, J., Liu, D., Cheng, Y., Liu, H., Yang, X., Wei, K., Zheng, Y., Jiang, X. \& Meng, L. 2018. Different responses of weather factors on hand, foot and mouth disease in three different climate areas of Gansu, China. BMC infectious diseases 18(1): 15.

[40] McCullagh, P. \& Nelder, J. A. 1989. Generalized Linear Models II. London: Chapman and Hall.

[41] Wood, S.N. 2006. Generalized additive models: an introduction with R. Chapman and Hall/CRC.

[42] Huang, Y., Deng, T., Yu, S., Gu, J., Huang, C., Xiao, G. \& Hao, Y. 2013. Effect of meteorological variables on the incidence of hand, foot, and mouth disease in children: a time-series analysis in Guangzhou, China. BMC infectious diseases 13(1): 134.

[43] Wei, J., Hansen, A., Liu, Q., Sun, Y., Weinstein, P. \& Bi, P. 2015. The effect of meteorological variables on the transmission of hand, foot and mouth disease in four major cities of Shanxi province, China: a time series data analysis (2009-2013). PLoS Negl Trop Dis 9(3): e0003572.

[44] Xiao, X., Gasparrini, A., Huang, J., Liao, Q., Liu, F., Yin, F., Yu, H. \& Li, X. 2017. The exposureresponse relationship between temperature and childhood hand, foot and mouth disease: a multicity study from mainland China. Environment international 100: 102-109.

[45] Liao, Y., Ouyang, R., Wang, J. \& Xu, B. 2015. A study of spatiotemporal delay in hand, foot and mouth disease in response to weather variations based on SVD: a case study in Shandong Province, China. BMC Public health 15(1): 71.

[46] Thanh, T.C. 2016. Effects of climate variations onhand-foot-mouth disease in Ho Chi Minh City. Vietnam Journal of Science and Technology 54(2A): 120.

[47] Hii, Y.L., Rocklöv, J. \& Ng, N. 2011. Short term effects of weather on hand, foot and mouth disease. PloS one 6(2): e16796.

[48] Tucker, P. \& Gilliland, J. 2007. The effect of season and weather on physical activity: a systematic review. Public health 121(12): 909-922. 